\title{
Tracking Multiple Objects Using a Kalman Filter and a Probabilistic Association Process
}

\author{
M. Marrón, J.C. García, M.A. Sotelo, F. Huerta, M. Cabello, J. Cerro \\ Electronics Department \\ University of Alcala \\ Alcalá de Henares, SPAIN \\ Email: marta@depeca.uah.es, sotelo@depeca.uah.es, jcarlos@depeca.uah.es
}

\begin{abstract}
In this paper one of the most important solutions in position estimation is used in conjunction with a data association algorithm in order to achieve a multi-tracking application. A Kalman Filter is extended and adapted in order to track the position and speed of a variable number of objects in an unstructured and complex environment. Both the developed algorithms and the results obtained with their real-time execution implementation in the mentioned application are described, and interesting conclusions extracted from these experiments are remarked in the paper. Finally, tracking results of the proposed algorithm are compared with another multi-object estimator based on a Particle Filter previously developed by the authors.
\end{abstract}

\section{INTRODUCTION}

Position estimation is one of the most important tasks in any robotic and intelligent vehicle application. This process is needed both to extract information about the robot environment and to know the concrete location of the robot itself. Taking into account the restrictions imposed by the measurements, the estimation process has to consider the noise related to them in order to achieve reliable information about the position. Thus, the algorithm has to include in the model this noisy behavior, generally by means of a probabilistic model. The main algorithms we can find in this context is the Kalman Filter (KF) [1].

In a multiple objects tacking task, the location process gets more complicated, as a measurement association is needed in order to run the estimator with the right input. The association problem is simplified if an only measurement for each object is in the measurement vector at each sample time. In contrast, the biggest the amount of information from each model is, the most reliable the estimation will be. In the work presented here visual information is used in order to obtain as more position information from each tracked object in the environment as possible. This fact leads to include a complex association algorithm with the estimation one, but increases the reliability of the tracking task.

Fig. 1 shows a functional diagram of the global tracking process. In the figure the image process used to obtain measurements of each object 3D position is shown. This algorithm is based on the information extracted from a pair of synchronized and digital B\&W cameras. The arrangement is statically mounted and calibrated in the autonomous robot in which the tracking task has to be done.

An algorithm based on the epipolar geometry of the vision system extract matching points in the objects edges in the two images captured at each sample time, and calculate the corresponding $3 \mathrm{D}$ position of these edge points in the robot environment. Edge points in the image (see Fig. 1) have been chosen to characterize the elements in the environment due to their robustness against light conditions and the simplicity of the vision algorithm used to extract and handle them.

This information is afterwards used by the position estimation process in order to track the objects related to the edge points. The stereo-vision process has been developed by the authors for this tracking application and is explained in detail in [2].

In this context different alternatives have also been tested by the research community, including Maximum Likelihood (ML), Nearest Neighbor (NN), Probabilistic Data Association (PDA), clustering, etc. In the work presented in this document the association algorithm used within the estimation process is the PDAF, as its probabilistic sound increases the robustness of the final estimation task.

In this paper, an adapted version of the $\mathrm{KF}$, developed in order to achieve the mentioned tracking objective is described in detail. Moreover, the conclusions extracted from the estimation results obtained with the proposed algorithm in the tracking task are presented.
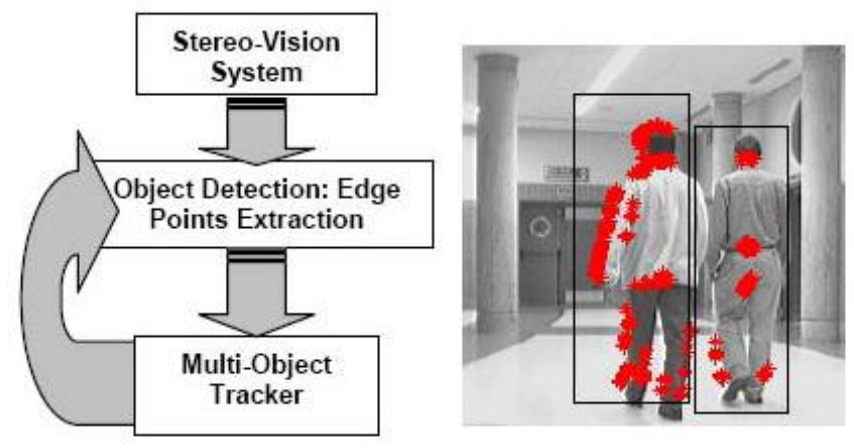

Fig. 1. Tracking process for multiple objects in indoor environments based on 3D position information extracted from a stereo-vision system. Red points in the image are edge points obtained from the objects detection process. 
Many proposals to solve the exposed problem, presented by the researching community imply the use of a Particle Filter (PF) as a multi-modal estimator ([3],[4],[5],[6]). The authors of this paper compare the results obtained with the proposed algorithm from the ones extracted from a PF based solution previously implemented ([7]).

\section{KALMAN FILTER as Position ESTIMATOR}

KF provides the optimal implementation of the Bayes Filter [1] when the system is linear and the measurements and model related noise is Gaussian with zero mean. This definition justifies the need of a more detailed analysis of the model to be used with the $\mathrm{KF}$ estimator.

A. The Estimation Process: The Kalmon Filter and the Position Model

The application of the $\mathrm{KF}$ requires a model definition in the terms shown in the following expression:

$$
\begin{aligned}
& \hat{x}_{k}=G \cdot \vec{x}_{k 1}+H \cdot \vec{u}_{k 1}+\vec{q}_{k 1} \\
& \vec{y}_{k}=C \cdot \hat{x}_{k}+\vec{y}_{k} / \hat{y}_{k}=C \cdot \hat{x}_{k}
\end{aligned}
$$

Applying the previous definition to the estimation problem of interest:

$\hat{x}_{k}=\left[\begin{array}{l}\hat{X}_{k} \\ \hat{Z}_{k}\end{array}\right]=\left[\begin{array}{ll}1 & 0 \\ 0 & 1\end{array}\right]\left[\begin{array}{l}\hat{X}_{k 1} \\ \hat{Z}_{k, 1}\end{array}\right]+\left[\begin{array}{cc}T_{s} & 0 \\ 0 & T_{s}\end{array}\right]\left[\begin{array}{c}v_{X, k 1} \\ v_{Z, k 1}\end{array}\right]+\vec{q}_{k 1}$,

where $\vec{y}_{k}=\left[\begin{array}{ll}X_{k} & Z_{k}\end{array}\right]$ is directly related with the state vector $\hat{x}_{k}$, and the state equation input vector is obtained from $\vec{y}_{k}$ and $\vec{x}_{k}$, as:

$$
\vec{u}_{k}=\frac{\vec{y}_{k} \quad \hat{x}_{k 1}}{T_{s}}
$$

In (2), $\left[\begin{array}{ll}X & Z\end{array}\right]^{\prime}$ are the Cartesian coordinates of the object centroid in the moving plane.

These model restrictions seem to be very restrictive in the use of the probabilistic estimator in different applications, but in fact the $\mathrm{KF}$ is systematically used in all type of estimation problems.

- Though the preliminary implementation of the $\mathrm{KF}$ is defined for continuous time, a discrete version of this estimator can be easily extracted, as demonstrated in [1]. In the same reference it is also asserted that the model linearity restriction specified in the $\mathrm{KF}$ definition can also be solved applying the extension of the algorithm. As the model previously defined for the application of interest is linear, a standard KF can be used in this case.

- The Gaussian character of the model noises is difficult to assert for most of estimation problems, as is the case of the one defined here. If this characteristic cannot be assured in the chosen model, the optimal estimation solution guaranteed by the KF will not be completed, and convergence problems in the estimation loop can appear. The convergence problem has not appeared in the estimation process developed in this work.

- The covariance matrixes $Q$ and $\mathrm{R}$ that respectively identify the noise vectors $\vec{q}$ and $\vec{p}$ in the model, should be diagonal in order to apply a state estimation process for this model with a $\mathrm{KF}$. This assessment cannot be easily theoretically assured in most of cases. For the model definition previously presented, an empirical study has been developed in order to obtain these two matrixes, whose result shows that the diagonal approximation can be applied in this case.

The KF functionality is shown in Fig. 2, where the two main steps of the probabilistic estimator can be seen. A detailed description of them can be found in [1].

Applying KF to the multi-tracking problem that presents the application of interest imposes some other problems. As the object dynamics changes from one object to other, $\mathrm{KF}$ can only be used to track one object. It is possible to use an only estimator for all the objects if the state vector $\vec{x}_{k}$ size is dynamically adapted to include the state variables of objects that appear or disappear, but this is difficult to implement.

Different solutions proposed by the researching community with the mentioned idea can be examined in [8] and [9].

Thus, most solutions of the same multi-estimation problem through a $\mathrm{KF}$ are solved using one $\mathrm{KF}$ for each estimation to do, which means for each object to track (see [10], [11] and [12]).

In any case, as explained in the introduction paragraph, an association algorithm is needed in order to develop correctly the correction step of the algorithm

\section{B. The Association Process: The PDAF and its Insertion in the} Kalmon Filter

The Probabilistic Data Association Filter (PDAF) is a Bayesian approach to solve the data association problem exposed.

In the association process, the influence or probability of every candidate measurements to every object $\left(\beta_{i, j}\right)$ is calculated. To obtain an assignation result, only those measurements whose influence is above a threshold (also called gate) are related to each object.

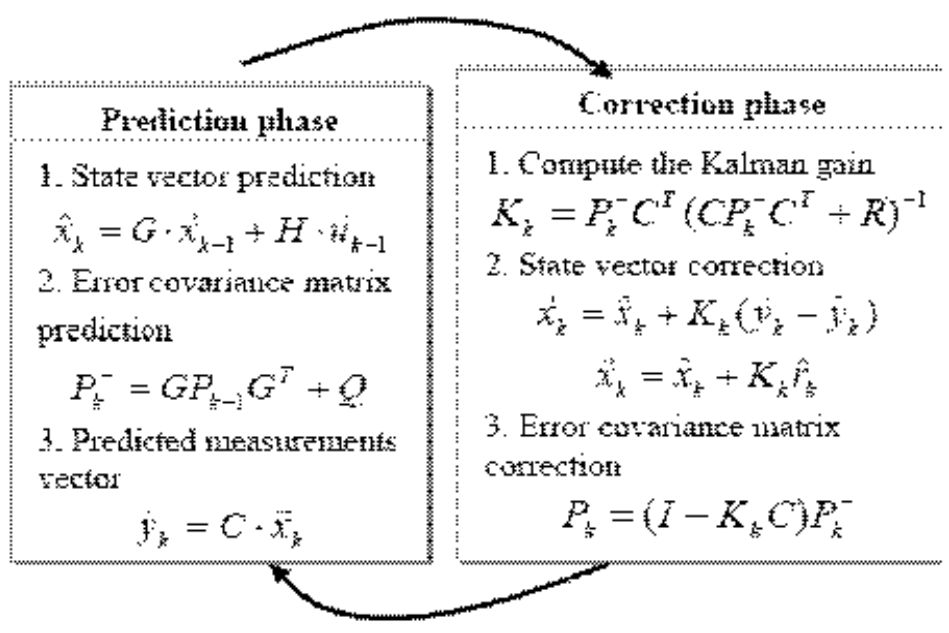

Fig.2. The Kalman Filter (KF) functionality. 
Thus, each $\beta_{i, j}$ is the probability of the association event $\theta_{i, j}$ that the ith measurement is originated by the object $j$ th ([13]). These events encompass all possible interpretations of the data, so that:

$$
\sum_{j=1}^{n} \beta_{i, j}
$$

where $n$ is the number of objects being tracked.

This probability can be calculated according to different parameters. In the work presented the characteristic chosen is the Euclidean distance from the $i t h$ measurement to the $j t h$ object predicted measurement vector $\hat{y}_{i}\left(d_{i, j}\right)$ :

$$
\beta_{i, j}=e^{-1 / 2 \frac{\min \left(d_{i, j}^{2}\right)}{R}} / j=1: m, j=1: n, d=\left|\vec{y}_{i}-\hat{y}_{j}\right|,
$$

where $m$ is the number of measurements extracted from the environment at each iteration.

If the distance to the closest predicted position is larger than the validation gate, the measure is associated to a new object, and a new KF is created in order to track it. This way, the tracking algorithm adapts on-line to estimate the position of a variable number of objects.

The validation gate is also used when calculating the association probability $\beta_{i, j}$ of each measurement ith to each object $j h$. This parameter is called $R$ in (5).

When applying a $\mathrm{PDAF}$ to a $\mathrm{KF}$, the calculation of the state vector corrected value in the correction step is modified, changing the standard innovation parameter $\left(\gamma_{j}\right)$ by the combined one:

$$
\gamma_{j}-\sum_{i=1}^{m} \beta_{i, j}
$$

The combined innovation is computed over the $m$ measurements assigned at each iteration to the $j$ th track

The final structure of the estimation algorithm that combines the $\mathrm{KF}$ and the PDAF can be seen in Fig. 3.

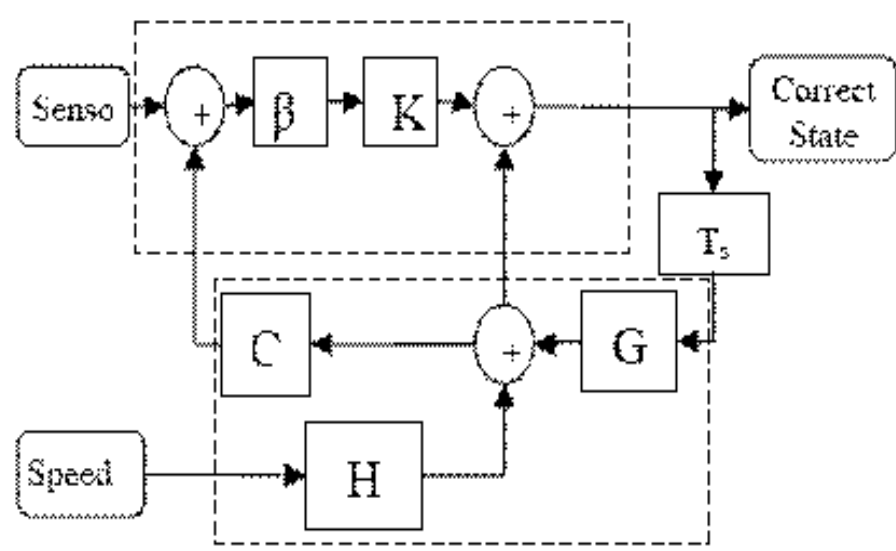

Fig. 3. Block diagram showing the functionality of the menlti-tracker designed, including the PDAF in the KF loop.

\section{RESULTS}

The algorithm has been run on a real-time system, mounted on board of a commercial robotic platform (Pioneer 2AT).

The sensory system (a pair of $B$ \&W digital and synchronized cameras) has been statically mounted and calibrated on top of the robot, at a height of $1.7 \mathrm{~m}$.

The stereo-vision runs in the same processing platform, generating position information of the obstacles in the environment at a 15 feps rate.

In this situation, different tests have been developed showing the correct functionality of the tracker designed, within various environmental conditions.

One up to six static and dynamic objects have been tracked with the proposed algorithm in videos of almost 2 minutes length (1653 frames). A value of $0.85 \mathrm{~m}$ has been used for the validation gate, and different types of static (persons and a robot) and static objects (a small trash, a tall box, etc.) area correctly tracked in almost all situations. A missing error rate of less than a $15 \%$ is achieved in complex situations (due to occlusions and sudden changes of the objects moving direction most of times), while tracking an only person and a static object gives a result free of errors in the position estimation task.

Comparing these results with the ones obtained with a Particle Filter (PF), developed by the authors in [7], this last algorithm gives better results (lower error rate), but has a slightly bigger execution time (10-20ms of the $K F$ versus 35 $65 \mathrm{~ms}$ of the $\mathrm{PF}$ ) runuing both algorithms in the same platform.

Fig. 4 shows the results generated by the designed tracker in a complex situation, in which five objects are being tracked in their movement all around the environment. A colored square has been drawn surrounding each tracked object within the gating distance.

This experiment shows the behavior of the tracking algorithm when a new object appears in the scene, and a new $\mathrm{KF}$ has to be initialized

\section{CONCLUSIONS}

In this paper, a KF is jointly used with a PDAF in order to implement a multiple object tracker. The functionality of the algorithm has been described, and its reliability has been demonstrated, in different environmental situations.

This algorithm has been developed in order to compare its behavior with a PF, described by the authors in [7]. This last algorithm seems to be more reliable than the $K F$, but shows a bigger execution time and complexity. These last arguments are the reasons for the author to conclude that $\mathrm{PF}$ may be more adequate for multi-tracking tasks in complex situations and the $\mathrm{KF}$ in should be the chosen solution in simpler ones are surveillance applications in low populated areas.

\section{ACKNOWLEGMENT}

This work was supported by the Ministry of Science and Technology under RESELAI project (reference TIN200614896-C02-01). 

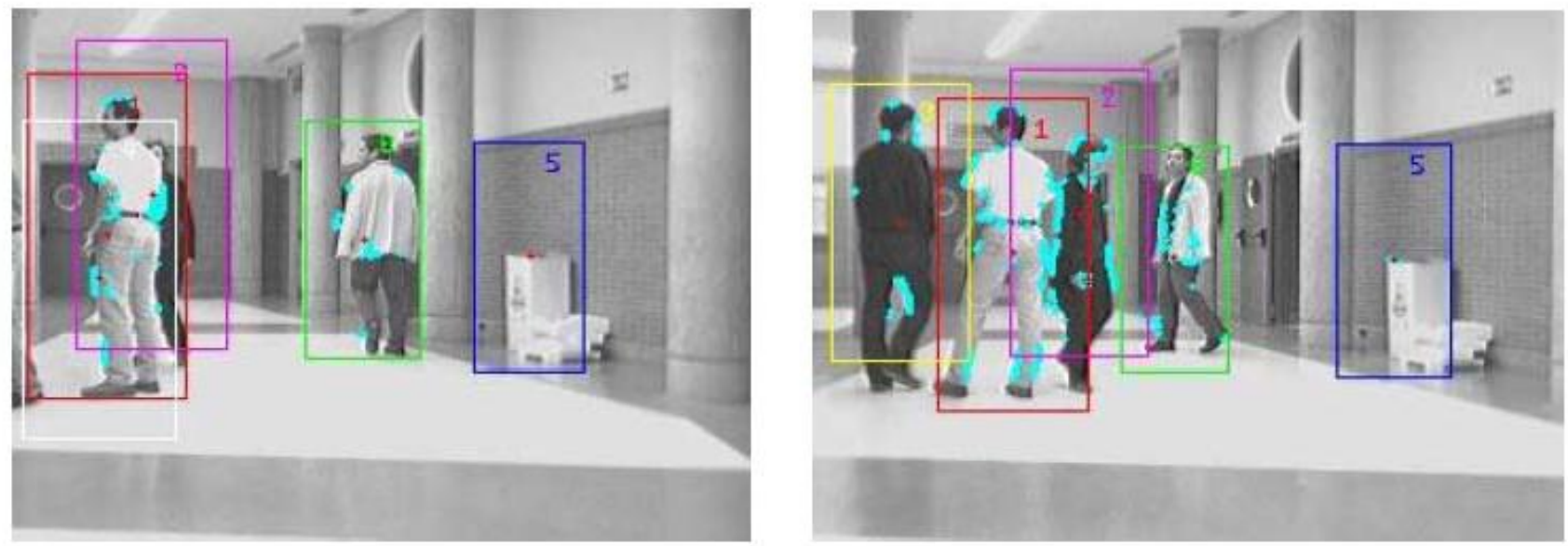

Fig. 4. Results of the position estimator developed in a complex situation, where five objects are breing tracked in their movements around the environment.

\section{REFERENCES}

[1] G. Welch, G. Bishop, "An Introduction to the Kalman Filter", ACM Inc., 2001 .

[2] M. Marron, M.A. Sotelo, Y.C. Garcia, D. Femandez, Y. Parra, "3D-Visual Detection of Multiple Objects and Environmental Structure in Complex and Dynamic Indoer Environments", Proceedings of the 32nd Anmuat Conference of the IEEE Industrial Electronics Saciety (IECONOO), ISBN: 1-4244-0136-4, pp. 3373-3378, Paris, November 2006.

[3] L.Y. Pao, R.M. Powers, "A Comparison of Several Different Approaches for Target Tracking with Clutter", Deparment of Cobrado at Boulder, USA, 2002 .

[4] D. Schulz, W. Burgard, D. Fox, A.B Cremers. "Tracking multiple moving targets with a mobile robot using particle filters and statistical data association", In Proc. of the IEEE Intemational Confereno on Robrotics \& Automation (CRR), 2001.

[5] C. Hue, Y.P. Le Cadre, P. Pèrez. "A particle filter to track multiple objects", IEEE Transactions on Aerospaøe and Electronic Systems, Vol. $38, \pi^{\circ} 3$, pp. $79 j-812$, July 2002 .

[6] D. Twoed, A. Calway. "Tracking Many Objects Using Subrrdinated Condensation", Proceedings of the British Machine Vision Conference (BMVC02), ISBN: j901725j97, pp. 283-292, October 2002 .

[7] M. Marnón, Y.C. García, M.A. Sotelo, D. Fernandez, D. Pizarro. "XYYFCP: An extended particle filter for tracking multiple and dynamic objects in complex environments", Prooedings of the IEEE International
Symposium on Industrial Electronics 2005 (ISIE05), ISBN: 0-78038738-4. Vol. I-rV, pp. 1587-1593, Dubrovnik, June 2005.

[8] D.B. Reid, "An algorithm for tracking multiple targets", IEEE Transactions on Automatic Control, Vol. 24, $\pi^{\circ}$ 6, pp. 843-854, December 1979.

[9] T. Schnitt, M. Bectz, R. Hanek, S. Buck. "Watch their moves applying probabilistic multiple object tacking to autonomous robot socoer", Proceedings of the Eighteenth National Conference on Artificial Intelligence (AABI02), ISBN: 0-262-5j329-0, pp. 599-604, Edmonton, July 2002 .

[10] Y.Y. Cox, S.L. Hingorani. "An efficient inplementation of Reid's multiple hypothesis tracking algorithm and its evaluation for the purpose of visual tracking", IEEE Transactions on Pattern Analysis and Machine Intelligence, vol. $18, \pi^{\circ} .2$, pp. $138-150, j 996$.

[ji] J. MacConnick, A, Blake. "A probabilistic exclusion principle for tracking multiple objects", Propeedings of the Seventh IEEE Intemational Conferenoe on Computer Vision (ICCV99), Vol. 1, pp. 572578, Corfil, September 1999.

[12] K. Smith, D. Gatica-Perez, J.M. Odobez. "Using particles to tack varying numbers of interacting people", Prowe dings of the Fourth IEEE Confereno on Computer Vicion and Pattern Recognition (CVPR0 05), pP. 962-969, San Diego, June 2005.

[13] C. Rasmussen, G.D. Hager, "Probabilistic Data Association Methods for Tracking Multiple and Compound Visual Objects", IEEE Transactions on Patern dnatysis and hachine Intelligence, $v 01.23, n^{\circ}$, pp. 560-576, 2001 . 